\title{
Circuit Quantum Electrodynamics with Carbon-Nanotube-Based Superconducting Quantum Circuits
}

\author{
Matthias Mergenthaler®, ${ }^{1,2, *, *}$ Ani Nersisyan, ${ }^{1}$ Andrew Patterson, ${ }^{1}$ Martina Esposito $\odot,{ }^{1}$ \\ Andreas Baumgartner $\odot,{ }^{3}$ Christian Schönenberger, ${ }^{3}$ G. Andrew D. Briggs, ${ }^{2}$ Edward A. Laird $\odot,{ }^{4,2}$ and \\ Peter J. Leek ${ }^{1, \dagger}$ \\ ${ }^{1}$ Clarendon Laboratory, Department of Physics, University of Oxford, Oxford OX1 3PU, United Kingdom \\ ${ }^{2}$ Department of Materials, University of Oxford, Oxford OXI 3PH, United Kingdom \\ ${ }^{3}$ Department of Physics, University of Basel, Klingelbergstrasse 82, Basel CH-4056, Switzerland \\ ${ }^{4}$ Department of Physics, Lancaster University, Lancaster LA1 4YB, United Kingdom
}

(Received 19 August 2020; revised 23 April 2021; accepted 20 May 2021; published 21 June 2021)

\begin{abstract}
Hybrid circuit QED involves the study of coherent quantum physics in solid-state systems via their interactions with superconducting microwave circuits. Here we present a crucial step in the implementation of a hybrid superconducting qubit that employs a carbon nanotube as a Josephson junction. We realize the junction by contacting a carbon nanotube with a superconducting $\mathrm{Pd} / \mathrm{Al}$ bilayer, and implement voltage tunability of the quantum circuit's frequency using a local electrostatic gate. We demonstrate a strong dispersive coupling to a coplanar waveguide resonator by investigating the gate-tunable resonator frequency. We extract qubit parameters from spectroscopy using dispersive readout and find qubit relaxation and coherence times in the range of 10-200 ns.
\end{abstract}

DOI: 10.1103/PhysRevApplied.15.064050

\section{INTRODUCTION}

Circuit quantum electrodynamics (cQED) with superconducting circuits [1] is a powerful platform used in on-chip quantum optics and quantum-information processing [2]. Hybrid superconducting circuits provide access to coherent quantum properties of other systems based on their interactions with microwave photons or artificial atoms [3-5]. In recent years, a variety of hybrid superconducting qubits have been realized by replacing the conventional aluminium $(S-I-S)$ Josephson junctions (JJs) with semiconductor-based $(S-N-S)$ JJs, such as InAs nanowires [6,7], (In, Ga)As heterostructures [8] and graphene $[9,10]$. For these $S-N-S$ JJs the normal- or semiconductor is contacted with a superconducting material enabling a supercurrent to flow due to the superconducting proximity effect [11]. Cooper-pair transport in such devices is described by Andreev reflections [12-14]. The conductance of semiconductors can be adjusted by applying a voltage to a nearby gate electrode, which tunes the Cooper-pair transport and hence the Josephson energy of the junction.

A strong technical motivation for these semiconductorsuperconductor hybrid JJ qubits is to realize gate-voltage

\footnotetext{
*mme@zurich.ibm.com

†peter.leek@physics.ox.ac.uk

$\ddagger$ Present address: IBM Quantum, IBM Research - Zurich, 8803 Rueschlikon, Switzerland
}

tunable qubits and hence eliminate decoherence due to magnetic flux noise. Further, electric fields are much easier to localize compared with magnetic fields, which makes complex multiqubit devices simpler to engineer. Additionally, qubit operation in moderate magnetic fields, for example, to explore interactions with different spin systems, can be made possible due to the robustness of these hybrid JJs to magnetic field $[9,15]$.

An interesting material to use in a $\mathrm{JJ}$ is the carbon nanotube (CNT), which can display ballistic electronic transport, and clean signatures of Andreev reflections when contacted with superconductors [16]. Using a CNT as the junction allows the use of its exceptional mechanical properties, which could offer a potential platform for creating quantum interference between a qubit and mechanical motion [17]. Further, ultraclean CNTs offer ballistic transport characteristics [18], which could provide JJs with lower defect density as opposed to conventional Al JJs with an amorphous tunnel barrier. This might have a potential positive impact on qubit coherence via elimination of two-level fluctuator defects in the amorphous tunnel barrier oxide [19-21]. Recent progress in CNT fabrication techniques might allow a CMOS-like design flow and processing of CNT JJs, which are free of nanoscale imperfections [22]. Hybrid devices incorporating proximitized CNTs allow the study of Andreev levels [16,23-25] and they are also predicted to carry Majorana fermions [26-28], which could be beneficial for topological quantum computing [29]. 
In the work presented here proximitized CNTs are used as the $\mathrm{JJ}$ in a common planar two-dimensional superconducting qubit architecture and their performance as a qubit is analyzed via a coupled microwave resonator. Resonator and qubit spectroscopy are performed as a function of applied gate voltage and strong dispersive coupling on the order of $100 \mathrm{MHz}$ is observed. Power-dependent qubit spectroscopy is used to extract the likely Josephson energy $E_{J}$ and transmission $T$ of the qubits' CNT junctions within a simple few-channel junction model [30]. Further, tentative evidence of Rabi oscillations is observed. Hence, the coherence is investigated using a pulse-chopping technique $[31,32]$ and qubit spectroscopy $[33,34]$, allowing $T_{1}$ and $T_{2}$ times in the range of 10-200 ns to be observed.

\section{CARBON-NANOTUBE-SUPERCONDUCTING QUANTUM CIRCUIT}

Figure 1 shows images and a circuit diagram of the device studied. Device fabrication begins with CNTs grown via chemical vapor deposition on a $\mathrm{Si} / \mathrm{SiO}_{2}$ $(450 \mu \mathrm{m} / 300 \mathrm{~nm})$ substrate. The microwave resonators are then patterned via electron-beam lithography (EBL). Prior to metal deposition an oxygen plasma etch is carried out to remove any CNTs that might electrically short the microwave circuits. Afterwards, $100 \mathrm{~nm}$ of $\mathrm{Al}$ is deposited via electron-beam evaporation. Following lift off, SEM imaging is used to locate and select CNTs for the qubits. The contacts to the CNT and island of the qubit are then patterned with EBL, postdevelopment cleaned using UV ozone and metalized with a Pd/Al $(4 / 80 \mathrm{~nm})$ bilayer. Before sample mounting, the room-temperature resistances of the CNT JJs are measured to check the fabrication yield. Roughly $80 \%$ of the fabricated devices conduct at room temperature and exhibit resistances between 7 $\mathrm{k} \Omega<R_{n}<100 \mathrm{k} \Omega$. A series of three chips are fabricated, each consisting of ten potential qubits. Each chip contains $10 \lambda / 4$ resonators with different frequencies, multiplexed via capacitive coupling to a single microwave transmission line [Fig. 1(a)]. Close to each resonator's electric field antinode, qubits are fabricated, and a dedicated dc electrostatic gate is used for control of the chemical potential of the CNT [Figs. 1(b) and 1(c)].

\section{CIRCUIT SPECTROSCOPY}

The system is cooled below $20 \mathrm{mK}$ in a dilution refrigerator and measured using standard cQED measurement techniques, see Fig. 1(d). First, the transmission spectrum of a device is measured via the feedline to identify the individual resonance frequencies of the ten resonators, at each of which a narrow (approximately $1 \mathrm{MHz}$ ) absorption dip is observed. Subsequently, $S_{21}$ spectroscopy as a function of gate voltage $V_{G}$ of each individual resonance is performed and resonators that exhibit a clear gate-dependent
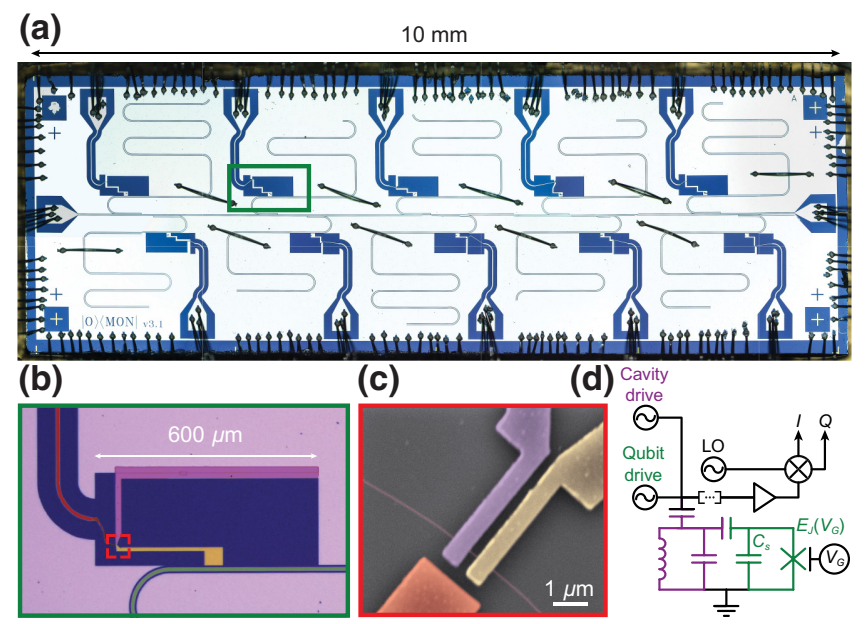

(c)

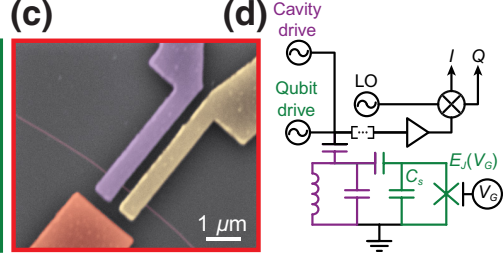

FIG. 1. Carbon-nanotube-superconducting quantum-circuit device. (a) Optical image of the device chip. A coplanar microwave transmission line in the center addresses ten multiplexed $\lambda / 4$ resonators with different resonance frequencies. Each resonator has a cut out in the ground plane close to its electric field antinode for qubit fabrication and a single dc line allowing voltage tunability of the qubit frequency. (b) False-color optical image of a single qubit with the island (yellow) capacitively coupled to the resonator (green) and the other side shorted to the ground plane (purple). The island capacitively shunts the CNT JJ to the surrounding ground plane. A side gate (red) is used to tune the circuit's frequency. (c) False-color SEM image of a CNT (pink) contacted with two superconducting contacts (yellow and purple) separated by $300 \mathrm{~nm}$ and a side gate (red). (d) Electrical circuit diagram of the device along with a sketch of its readout and control circuitry. The qubit (green) is capacitively coupled to the resonator (purple). A side gate with applied voltage $V_{G}$ tunes the Josephson energy $E_{J}$ of the qubit. The resonator itself is capacitively coupled to a transmission line, which is used to send microwave tones to the qubit and its response is measured using a standard heterodyne detection scheme.

resonance frequency are selected for further investigations as these potentially correspond to working CNT qubits. Usually $20-50 \%$ of all devices on one chip show this dependence. From these, two devices showing similar gate-dependent behavior are carefully characterized, hereafter labeled as device QA and QB.

Figure 2(a) shows resonator spectroscopy as a function of dc voltage applied to the gate electrode $\left(V_{G}\right)$ on device QA. At a single gate voltage $V_{G}$, an absorption line corresponding to the resonator is observed [inset Fig. 2(b)]. Tuning $V_{G}$ the absorption line moves in frequency, exhibiting a broad gate region $\left(V_{G}<-30 \mathrm{~V}\right)$ where it is approximately constant, indicating the bare resonator frequency of $f_{0}=5.572 \mathrm{GHz}$, and another region $\left(V_{G}>-20\right.$ $\mathrm{V})$ where the resonance is quasiperiodically shifting to frequencies up to approximately $10 \mathrm{MHz}$ higher than $f_{0}$. In cQED this is indicative of the resonator being dispersively coupled to a circuit with tunable transition frequency $f_{Q}<$ 


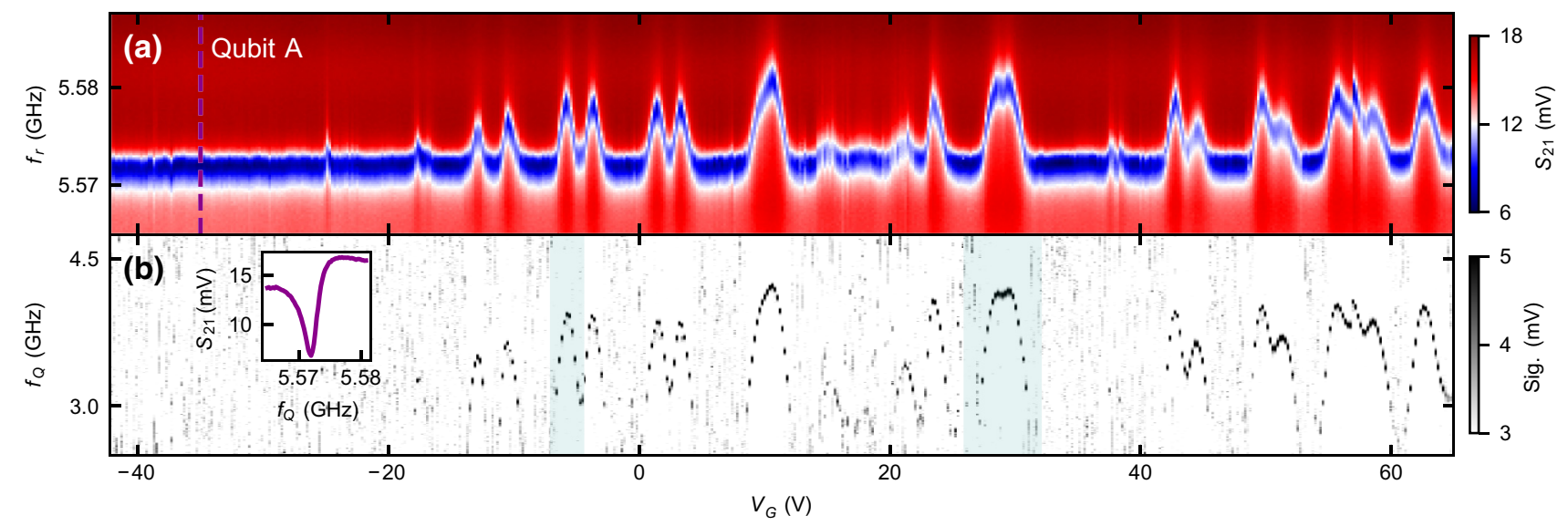

FIG. 2. Resonator and qubit spectroscopy as a function of applied gate voltage $V_{G}$ for device QA. The transmission $S_{21}$ is expressed in terms of the corresponding demodulated signal voltage. (a) Resonator spectroscopy of device QA as a function of $V_{G}$. (b) Qubit spectroscopy of device QA as a function of $V_{G}$. The shaded areas mark the features used for qubit analysis (cf. Fig. 3, $V_{G}$ is shifted due to drift). Inset: line-cut through spectrum indicated in (a).

$f_{0}$. From here onwards we refer to the circuit as qubit and investigate the circuit's level of qubit behavior.

In a second measurement, we carry out spectroscopy to identify the potential qubit's frequency. Here the cavity drive is set to track the particular resonance frequency $f_{r}$ at each $V_{G}$ and simultaneously a spectroscopic probe tone is fed onto the input line (qubit drive). While the qubit drive is swept in frequency the amplitude response at $f_{r}$ is measured. This measurement performed on device QA is presented in Fig. 2(b). A spectroscopic response is observed for $V_{G}>-30 \mathrm{~V}$. The values of $V_{G}$ exhibiting a spectroscopic response coincide exactly with the values exhibiting shifts in $f_{r}$, cf. Fig. 2(a). This is consistent with the dispersive regime of cQED [35] indicating the presence of a qubit, frequency tunable between $2.8 \mathrm{GHz}<f_{Q}<4.2$ GHz. The data in Fig. 2(b) likely shows the $f_{01}=f_{Q}$, i.e., ground to first excited state transition of the qubit as a function of gate voltage. Similar measurements are also performed on device QB, see the Supplemental Material [36] and Table I.

We begin our analysis by using a simple model of a twolevel system dispersively coupled to a harmonic oscillator. In this model the coupling strength $g$ between the resonator and the qubit can be estimated using the dispersive shift $\chi$ of the resonator $[33,37]$. To first order, due to the coupling to the $f_{01}$ transition, $\chi=\left(g^{2} / \Delta\right)$, where $\Delta=f_{r}-f_{Q}$ is the detuning between the qubit and the resonator [37]. Using

TABLE I. Parameters extracted from resonator and qubit spectroscopy measurements for devices QA and QB.

\begin{tabular}{lcccc}
\hline \hline & $f_{0}[\mathrm{GHz}]$ & $f_{Q}[\mathrm{GHz}]$ & $g_{\max }[\mathrm{MHz}]$ & $\chi_{\max }[\mathrm{MHz}]$ \\
\hline Qubit A & 5.572 & $2.8-4.2$ & 113 & 10 \\
Qubit B & 4.595 & $2.4-3.5$ & 85 & 7 \\
\hline \hline
\end{tabular}

this expression and the data of QA [Figs. 2(a) and 2(b)] yields an estimate of the coupling strength in the range $48<g<113 \mathrm{MHz}$ that increases with observed $f_{Q}$, see the Supplemental Material [36]. This behavior is expected for transmon qubits because $g \propto E_{J}^{1 / 4}$ and $f_{Q} \propto E_{J}{ }^{1 / 2}$ [37]. Similar values are observed with $\mathrm{QB}$, summarized in Table I.

\section{QUBIT CHARACTERIZATION}

In reality our qubits are not expected to be pure twolevel systems but rather anharmonic oscillators with many levels [37], and hence exhibit more than one transition. Therefore, mapping out $f_{01}$ is the first step of characterizing the voltage tunable qubit. To fully describe the qubit, the Josephson energy $E_{J}$, charging energy $E_{C}$, transmission $T$, and the number of conduction channels $N$ are needed. Electrostatic finite-element simulations for the exact qubit designs yield values for the shunt capacitance to ground and the island's capacitance to the resonator [cf. Fig. 1(b)], both contributing to the overall capacitance $C_{\Sigma}$ necessary to calculate $E_{C}=e^{2} / 2 C_{\Sigma}\left(E_{C}{ }^{Q A}=508 \mathrm{MHz}, E_{C} Q B=\right.$ $391 \mathrm{MHz}$ ). Nonetheless, the other parameters related to the JJ cannot be deduced from the qubit's fundamental frequency alone. Here, we show that they can be extracted by measuring the qubit at different drive powers. For this purpose, we use the same qubit spectroscopy technique as in Fig. 2(b), holding $V_{G}$ fixed and varying the qubit drive power. Such a measurement is presented in Fig. 3(a). At a low drive power, $P=-45 \mathrm{dBm}$ at the output of the microwave generator, only a single peak is observed in the qubit spectroscopy [Fig. 3(a) bottom trace]. If the power of the qubit drive is increased to $P=-30 \mathrm{dBm}$, a more complicated multipeak response is observed, which exhibits a second peak at frequencies just below $f_{01}$, see Fig. 3(a). 

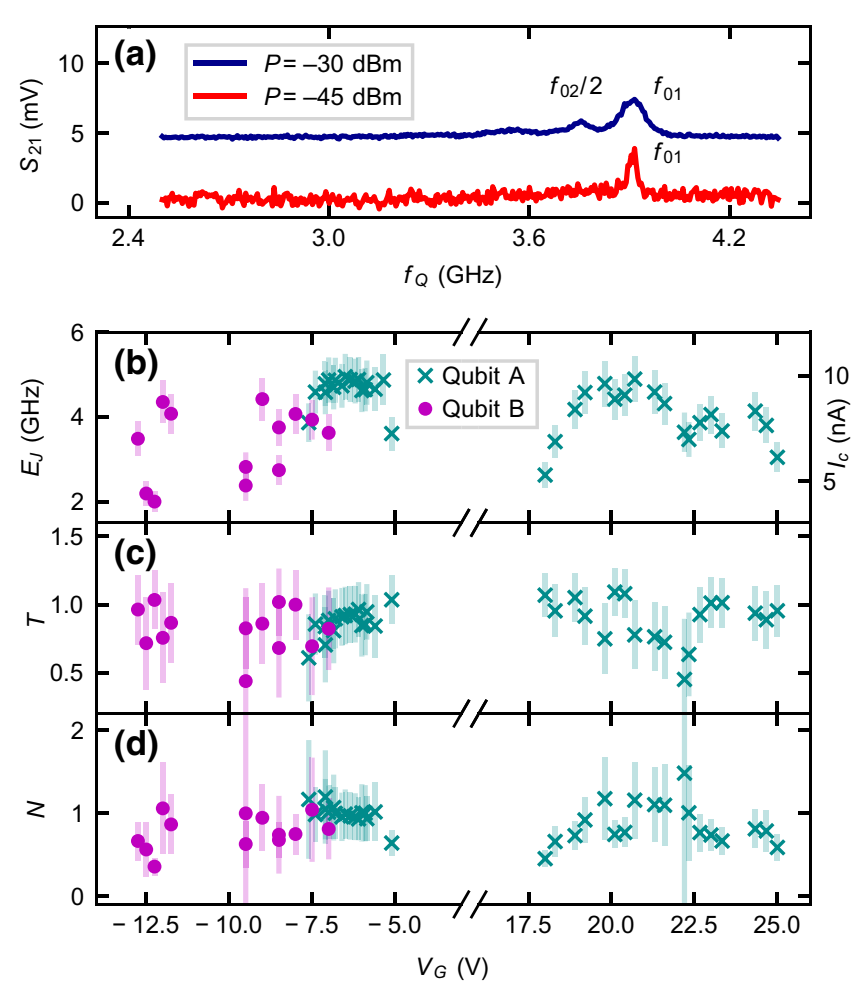

FIG. 3. Qubit spectroscopy, qubit parameter $E_{J}$, and junction characteristics $T$ and $N$. (a) Qubit spectroscopy traces at two different qubit drive powers for device QA, offset for clarity. (b) Extracted values for $E_{J}$ as a function of $V_{G}$ for QA (cyan crosses) and QB (purple points). The critical current $I_{c}$, calculated from $E_{J}$ is indicated on the right $y$ axis. (c) Extracted values for $T$ as a function of $V_{G}$ for QA and QB. (d) Values of $N$ calculated from $T$.

This is indicative of a weakly anharmonic circuit, such as a transmon qubit, where the lower peak corresponds to the two-photon transition from the ground state to the second excited state, i.e., $f_{02} / 2$. Note that this second spectral peak is not always clearly present in our data on these devices, and a broader spectral feature is consistently seen at higher drive powers, which is not possible to resolve into clear individual peaks. This may be due to significant charge dispersion of higher qubit energy levels, or other sources of decoherence.

We measure the frequency of the two clearest spectral lines over a range of $V_{G}$ [indicated in Fig. 2(b)] from data similar to that seen in Fig. 3(a). Interpreting them as the $f_{01}$ and $f_{02} / 2$ transitions of a qubit with at least three energy levels, we can extract a possible anharmonicity between these three levels as $\alpha=2\left(f_{01}-f_{02} / 2\right)$.

CNT JJs and other types of JJs, such as weak links made from narrow superconducting constrictions, normal metal, or a semiconductor, have energy phase relations that differ from standard $S-I-S$ JJs [38-41]. Assuming the CNT JJs with a channel length of $300 \mathrm{~nm}$ are in the short-junction regime, Cooper-pair transport is mediated by Andreev bound states [41-43]. Hence, we cannot interpret the measurements by using a standard Cooper-pair-box (CPB) Hamiltonian, but rather by using a second-order perturbation theory approach to a modified CPB Hamiltonian presented by Kringhøj et al. [30]. Here, $\alpha$ and $f_{01}$ are given by

$$
\begin{gathered}
h \alpha=E_{C}\left(1-\frac{3 T}{4}\right)-\frac{E_{C}^{3 / 2}}{\sqrt{2 E_{J}}}\left[1-\frac{15}{4} T\left(1-\frac{3 T}{4}\right)\right] \\
h f_{01}=\sqrt{8 E_{J} E_{C}}-E_{C}\left(1-\frac{3 T}{4}\right) \\
+\frac{E_{C}^{3 / 2}}{2 \sqrt{2 E_{J}}}\left[1-\frac{15}{4} T\left(1-\frac{3 T}{4}\right)\right]
\end{gathered}
$$

where it is assumed that all conduction channels exhibit equal transmission $T$, see the Supplemental Material for details [36]. Using Eqs. (1) and (2) together with the values extracted for $f_{01}, \alpha$ and $E_{C}$, the parameters $E_{J}$ and $T$ can be calculated by solving the resulting set of equations, yielding a deeper understanding of the qubit's CNT JJ.

In Fig. 3(b) the Josephson energy $E_{J}$, extracted with the method mentioned above, is presented as a function of gate voltage $V_{G}$ [note a small gate drift compared with Fig. 2(b)]. The error bars are determined by the measurement error of $f_{01}$ and $f_{02} / 2$, as the qubit power spectroscopy data can be noisy and sometimes exhibit a complicated multipeak structure, making the peak distinction difficult. The Josephson energy can also be used to calculate the critical current $I_{c}$ of the qubit's JJ using $E_{J}=\hbar I_{c} /(2 e)$, see Fig. 3 (b). We find $4 \mathrm{nA}<I_{c}<10 \mathrm{nA}$, with an average value $\left\langle I_{c}\right\rangle=8.2 \pm 1.6 \mathrm{nA}$ across both qubit devices. These values of $I_{c}$ are comparable to $1 \mathrm{nA}<I_{c}<17 \mathrm{nA}$ that we independently observed in dc bias spectroscopy of CNT JJs contacted with identical processing. The extracted values of $T$ are presented in Fig. 3(c). We consistently find $0.33<T<1$ with a mean value of $\langle T\rangle=0.85 \pm 0.16$, indicating high-quality contacts to the CNT. Further, the extracted values of $T$ can be used to calculate the number of channels $N$ contributing to transport via $E_{J}=\Delta N T / 4$ [30], where $\Delta$ is the induced superconducting gap of the CNT JJ (we estimate $\Delta=90 \pm 10 \mu \mathrm{eV}$ using dc bias spectroscopy measurements on CNT devices contacted with identical processing [44]), see Fig. 3(d). The calculated values for $N$ congregate around $N=1$, indicating that only one conduction channel is strongly coupled to the superconducting contacts. This is in strong contrast to conventional aluminium $S-I-S$ JJs, where $N \gg 1$ and $T \ll 1$.

The obtained values of $E_{J}$ in conjunction with the simulated values of $E_{C}$ can be used to calculate the ratio $E_{\mathrm{J}} / E_{\mathrm{C}}$. We find a mean $E_{\mathrm{J}} / E_{\mathrm{C}} \approx 9 \pm 2$ for both QA and 
QB. These values agree well with electrostatic simulations of the device design, yielding $E_{\mathrm{J}} / E_{\mathrm{C}} \sim 12$, giving confidence in the extraction method of $E_{J}$ and $T$. Note that the estimated average $E_{\mathrm{J}} / E_{\mathrm{C}}$ ratio places the qubit between the transmon regime $\left(E_{\mathrm{J}} / E_{\mathrm{C}}>20\right)$ and the Cooper-pairbox regime $\left(E_{\mathrm{J}} / E_{\mathrm{C}}<1\right)$, where a low $E_{\mathrm{J}} / E_{\mathrm{C}}$ leads to a complex energy-level structure with charge dispersion making the qubit susceptible to charge noise [37]. However, charge dispersion is predicted [45] and shown [46] to vanish in channels where the transmission is approaching unity, which is the case for JJs made from CNTs.

\section{RELAXATION AND COHERENCE}

We finally report on investigations of the relaxation and coherence times of the CNT-qubit devices. Timedomain measurements with a weak continuous readout drive [47,48] show evidence of Rabi oscillations [36]. In Fig. 4(a) the extracted Rabi oscillation frequency $\Omega$ is shown as a function of the qubit drive amplitude. For drive amplitudes $<0.5 \mathrm{~V}, \Omega$ increases linearly with a slope of approximately $78.3 \mathrm{MHz} / \mathrm{V}$, which is a characteristic feature of Rabi oscillations. For pulse amplitudes $>0.5 \mathrm{~V}$,
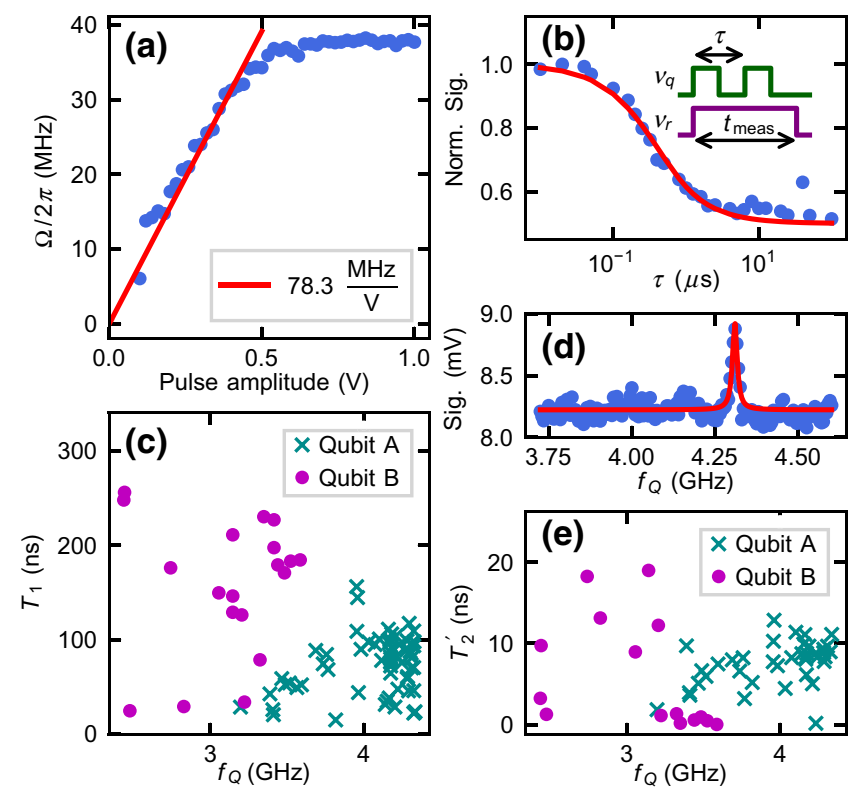
के 8
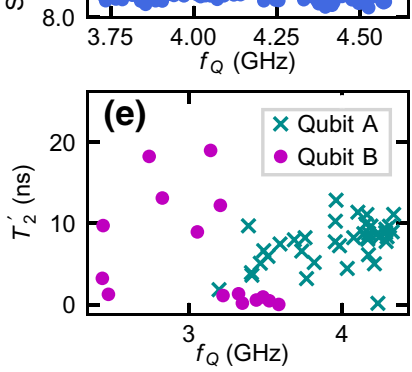

FIG. 4. Signatures of Rabi oscillations and $T_{1}$ and $T_{2}^{\prime}$ measurements. (a) Rabi-oscillation frequency $\Omega / 2 \pi$ as a function of the qubit pulse amplitude. For pulse amplitudes $<0.5 \mathrm{~V}, \Omega / 2 \pi$ is fit with a linear function (solid red line) yielding a slope of 78.3 $\mathrm{MHz} / \mathrm{V}$. (b) Data of a single $T_{1}$ experiment on device QA. The measurement response is fitted to Eq. (6) (red curve), yielding $T_{1}=117.3 \pm 5.8 \mathrm{~ns}$. Inset: pulse scheme (qubit pulse, green; measurement pulse, purple). (c) Measured $T_{1}$ as a function of $f_{Q}$ for devices QA (crosses) and QB (circles). (d) A single measurement of $T_{2}^{\prime}$ on device QA. Qubit spectroscopy trace is fitted to Eq. (7) (red curve), yielding $T_{2}^{\prime}=19.0 \pm 1.5 \mathrm{~ns}$. (e) Measured $T_{2}^{\prime}$ as a function of $f_{Q}$ for devices QA (crosses) and QB (circles).
$\Omega$ saturates at around approximately $38 \mathrm{MHz}$, which may be explained as being due to the low anharmonicity of the qubit, i.e., the qubit will be driven into higher-energy states at drive rates greater or equal to the anharmonicity. It is worth mentioning that the decay of the observed oscillations yielded a time constant of approximately 50 ns, which is consistent with the coherence measurements presented below. The experimental setup is not suitable to explicitly resolve such fast decaying Rabi oscillations. Hence, for early qubit analysis we employ an alternative pulsed technique for measuring the relaxation time, previously used in quantum-dot charge qubits [31,32]. The method is tested on conventional superconducting qubits to confirm that it yields the same result as standard techniques (see the Supplemental Material [36]).

To measure $T_{1}$, a pulse-chopping method [31,32] is used. The resonator is continuously measured with a weak cavity drive at $f_{0}$ for a time of $100 \mu \mathrm{s}$. Simultaneously, the qubit is driven on resonance with a pulse train of $50 \%$ duty cycle, and for each measurement the pulse period $\tau$ is varied, see inset Fig. 4(b). For very short $\tau$, i.e., $\tau \ll T_{1}$, the qubit drive randomizes the qubit between the ground and first excited state and it has no time to relax. In the case of very long $\tau$, i.e., $\tau \gg T_{1}$, the qubit has time to relax to the ground state in between drive pulses. Therefore, in the latter case, the measured signal is the time average of the qubit being in the ground and excited state, giving a signal of half the value found in the limit $\tau \rightarrow 0$. A measurement following this procedure is presented in Fig. 4(b). The data is normalized with respect to a measurement with the qubit drive turned off and fitted to

$$
S(\tau)=\frac{1}{2}+\frac{T_{1}\left(1-e^{-\tau /\left(2 T_{1}\right)}\right)}{\tau}
$$

where $T_{1}$ is the only free parameter. In the measurement shown in Fig. 4(b) the fit yields $T_{1}=117.3 \pm 5.8 \mathrm{~ns}$. The relaxation time $T_{1}$ is measured across a range of gate voltages, and therefore a range of $f_{Q}$, for both devices, see Fig. 4(c). QB on average exhibits a longer $\left\langle T_{1, Q B}\right\rangle=151 \pm 71$ ns than QA where $\left\langle T_{1, Q A}\right\rangle=74 \pm 30 \mathrm{~ns}$. The longest $T_{1}$ values are 250 and $150 \mathrm{~ns}$ for QB and QA, respectively.

A lower bound, $T_{2}^{\prime}$, on qubit coherence time $T_{2}$ can be found by measuring the linewidth of a low-power qubit spectroscopy trace [33,34], see Fig. 4(d). Fitting a Lorentzian with linewidth $2 \delta v_{\mathrm{HWHM}}$ to the qubit transition peak, $T_{2}^{\prime}$ can be calculated via

$$
2 \pi \delta v_{\mathrm{HWHM}}=\frac{1}{T_{2}^{\prime}}=\left(\frac{1}{T_{2}^{2}}+n_{s} \omega_{\mathrm{vac}}^{2} \frac{T_{1}}{T_{2}}\right)^{1 / 2},
$$

where $n_{s} \omega_{\text {vac }}^{2}$ is proportional to the microwave input power, $\omega_{\text {vac }}^{2}$ the vacuum Rabi frequency, and $n_{s}$ the number of photons in the resonator $[33,34]$. Hence, at low qubit drive powers the linewidth should be the least broadened. Here, 
low power corresponds to the lowest qubit drive power, which still results in a visible qubit spectroscopy peak. We assume this power corresponds to $n_{s} \approx 0$. However, it is worthwhile to note that we specifically quote $T_{2}^{\prime}$ as a lower limit for $T_{2}$ and hence $n_{s}$ does not need to be known. This results in $T_{2}^{\prime}=19.0 \pm 1.5 \mathrm{~ns}$ for the data presented in Fig. 4(d).

The measurement and its analysis is repeated for different qubit frequencies $f_{Q}$, see Fig. 4(e). While $T_{2}^{\prime}$ seems to increase slightly with increasing $f_{Q}$ for QA, this is not true for QB. Coherence is highest at around $f_{Q}=3 \mathrm{GHz}$, with $T_{2}^{\prime}=25 \mathrm{~ns}$ for $\mathrm{QB}$, but significantly reduced at $f_{Q}=3.5$ GHz. On average QA exhibits a longer $\left\langle T_{2, \mathrm{QA}}^{\prime}\right\rangle=10 \pm 5$ ns compared with $\mathrm{QB}\left\langle T_{2, \mathrm{QB}}^{\prime}\right\rangle=6 \pm 6 \mathrm{~ns}$. We stress that these coherence times represent only a lower bound for $T_{2}$.

Decoherence and fast relaxation in these devices could be attributed to dissipation due to dirty, disordered CNTs, Purcell decay into the gate line, strong dielectric loss due to the thick $\mathrm{SiO}_{2}$, and residual resistance to the superconducting leads. The loss tangent of thermal $\mathrm{SiO}_{2}$ is measured to be $\tan \delta \sim 3 e^{-4}$ [49], limiting the qubit's relaxation time to $T_{1} \sim 1 \mu \mathrm{s}$ within the accessible frequency range. Dissipation in nanoscale weak-link JJ oscillators, made from aluminium, was previously mentioned as a possible source of decoherence [50-52]. Additionally, the short $T_{2}^{\prime}$ could also stem from Andreev levels in the junction interacting with acoustic phonons $[25,53,54]$.

\section{CONCLUSION}

The experiments described here demonstrate crucial steps in the implementation of a voltage tunable superconducting qubit based on a CNT JJ. The device is of similar geometry and exhibits similar gate voltage behavior to previously reported voltage tunable superconducting qubit devices [6-10]. Simultaneous resonator and qubit spectroscopy show clear evidence of qubit-resonator coupling with coupling strength on the order of $g \sim 100 \mathrm{MHz}$, comparable to cQED experiments with conventional transmon qubits. Qubit spectroscopy at high drive powers is used to extract the qubit parameter $E_{J}$ and ratio $E_{J} / E_{C}$ as well as $I_{c}$ and $T$ of the CNT JJ. From the values for $T$, values of $N \sim 1$ are calculated, indicating that only one conduction channel is strongly coupled to the superconducting leads of the qubit. Further, evidence of Rabi oscillations as well as qubit relaxation and coherence times in the range 10-200 ns are observed. It remains to explicitly demonstrate Rabi oscillations and full qubit control.

Advances in fabrication, e.g., using suspended ultraclean CNT JJs could lead to significant improvements to the coherence times of these devices. Such JJs have already been individually realized [55]. Fabrication improvements in other hybrid qubit designs, such as those based on
InAs nanowires resulted in $T_{1}=5-20 \mu \mathrm{s}[15,56]$, similar to state-of-the-art, aluminium-based flux-tunable transmon qubits [57]. The implementation of a superconducting quantum circuit based on a CNT presented here offers potential for unique experiments in order to create quantum interference between a qubit and mechanical motion [17]. Additionally, CNT-based qubits could be used as ultrasensitive force sensors [58] and if arranged in a superconducting quantum interference device geometry as a detector for magnetic moments [38]. Furthermore, these qubits based on proximitized CNTs could be utilized to study Andreev physics [23-25] and investigate the prediction of carrying Majorana fermions [26-28], which could be valuable for topological quantum computing.

\section{ACKNOWLEDGMENTS}

We acknowledge support from the Royal Academy of Engineering, EPSRC (EP/R029229/1, EP/J001821/1 and EP/J013501/1), Swiss Nanoscience Institute (SNI), and the Swiss National Science Foundation. M.M. acknowledges support from the Stiftung der Deutschen Wirtschaft (sdw).

[1] A. Wallraff, D. I. Schuster, A. Blais, L. Frunzio, R.S. Huang, J. Majer, S. Kumar, S. M. Girvin, and R. J. Schoelkopf, Strong coupling of a single photon to a superconducting qubit using circuit quantum electrodynamics., Nature 431, 162 (2004).

[2] G. Wendin, Quantum information processing with superconducting circuits: A review, Rep. Prog. Phys. 80, 106001 (2017).

[3] Z.-L. Xiang, S. Ashhab, J. You, and F. Nori, Hybrid quantum circuits: Superconducting circuits interacting with other quantum systems, Rev. Mod. Phys. 85, 623 (2013).

[4] G. Kurizki, P. Bertet, Y. Kubo, K. Mølmer, D. Petrosyan, P. Rabl, and J. Schmiedmayer, Quantum technologies with hybrid systems, Proc. Natl. Acad. Sci. 112, 3866 LP (2015).

[5] A. Cottet, M. C. Dartiailh, M. M. Desjardins, T. Cubaynes, L. C. Contamin, M. Delbecq, J. J. Viennot, L. E. Bruhat, B. Douçot, and T. Kontos, Cavity QED with hybrid nanocircuits: From atomic-like physics to condensed matter phenomena, J. Phys.: Condens. Matter 29, 433002 (2017).

[6] G. de Lange, B. van Heck, A. Bruno, D. J. van Woerkom, A. Geresdi, S. R. Plissard, E. P. A. M. Bakkers, A. R. Akhmerov, and L. DiCarlo, Realization of Microwave Quantum Circuits Using Hybrid Superconducting-Semiconducting Nanowire Josephson Elements., Phys. Rev. Lett. 115, 127002 (2015).

[7] T. Larsen, K. Petersson, F. Kuemmeth, T. Jespersen, P. Krogstrup, J. Nygård, and C. Marcus, SemiconductorNanowire-Based Superconducting Qubit, Phys. Rev. Lett. 115, 127001 (2015).

[8] L. Casparis, M. R. Connolly, M. Kjaergaard, N. J. Pearson, A. Kringhøj, T. W. Larsen, F. Kuemmeth, T. Wang, C. Thomas, S. Gronin, G. C. Gardner, M. J. Manfra, C. 
M. Marcus, and K. D. Petersson, Superconducting gatemon qubit based on a proximitized two-dimensional electron gas, Nat. Nanotechnol. 13, 915 (2018).

[9] J. G. Kroll, W. Uilhoorn, K. L. van der Enden, D. de Jong, K. Watanabe, T. Taniguchi, S. Goswami, M. C. Cassidy, and L. P. Kouwenhoven, Magnetic field compatible circuit quantum electrodynamics with graphene josephson junctions, Nat. Commun. 9, 4615 (2018).

[10] J. I.-J. Wang, D. Rodan-Legrain, L. Bretheau, D. L. Campbell, B. Kannan, D. Kim, M. Kjaergaard, P. Krantz, G. O. Samach, F. Yan, J. L. Yoder, K. Watanabe, T. Taniguchi, T. P. Orlando, S. Gustavsson, P. Jarillo-Herrero, and W. D. Oliver, Coherent control of a hybrid superconducting circuit made with graphene-based van der waals heterostructures, Nat. Nanotechnol. 14, 120 (2019).

[11] H. Meissner, Superconductivity of contacts with interposed barriers, Phys. Rev. 117, 672 (1960).

[12] A. F. Andreev, Thermal conductivity of the intermediate state of superconductors, J. Exptl. Theoret. Phys. (U.S.S.R.) 20, 2222 (1965).

[13] P. de Gennes and D. Saint-James, Elementary excitations in the vicinity of a normal metal-superconducting metal contact, Phys. Lett. 4, 151 (1963).

[14] D. Saint-James, Elementary excitations in the vicinity of the surface separating a normal metal and a superconducting metal, J. Phys. 25, 899 (1964).

[15] F. Luthi, T. Stavenga, O. W. Enzing, A. Bruno, C. Dickel, N. K. Langford, M. A. Rol, T. S. Jespersen, J. Nygård, P. Krogstrup, and L. DiCarlo, Evolution of Nanowire Transmon Qubits and Their Coherence in a Magnetic Field, Phys. Rev. Lett. 120, 100502 (2018).

[16] J.-D. Pillet, C. H. L. Quay, P. Morfin, C. Bena, A. L. Yeyati, and P. Joyez, Andreev bound states in supercurrentcarrying carbon nanotubes revealed, Nat. Phys. 6, 965 (2010).

[17] K. Khosla, M. Vanner, N. Ares, and E. Laird, Displacemon Electromechanics: How to Detect Quantum Interference in a Nanomechanical Resonator, Phys. Rev. X 8, 021052 (2018)

[18] M. Jung, J. Schindele, S. Nau, M. Weiss, A. Baumgartner, and C. Schönenberger, Ultraclean single, double, and triple carbon nanotube quantum dots with recessed Re bottom gates, Nano Lett. 13, 4522 (2013).

[19] A. Shnirman, G. Schön, I. Martin, and Y. Makhlin, Lowand High-Frequency Noise from Coherent Two-Level Systems, Phys. Rev. Lett. 94, 127002 (2005).

[20] S. Oh, K. Cicak, J. S. Kline, M. A. Sillanpää, K. D. Osborn, J. D. Whittaker, R. W. Simmonds, and D. P. Pappas, Elimination of two level fluctuators in superconducting quantum bits by an epitaxial tunnel barrier, Phys. Rev. B 74, 100502 (2006)

[21] C. Müller, J. H. Cole, and J. Lisenfeld, Towards understanding two-level-systems in amorphous solids: Insights from quantum circuits, Rep. Prog. Phys. 82, 124501 (2019)

[22] G. Hills, C. Lau, A. Wright, S. Fuller, M. D. Bishop, T. Srimani, P. Kanhaiya, R. Ho, A. Amer, Y. Stein, D. Murphy, Arvind, A. Chandrakasan, and M. M. Shulaker, Modern microprocessor built from complementary carbon nanotube transistors, Nature 572, 595 (2019).
[23] J. Gramich, A. Baumgartner, and C. Schönenberger, Andreev bound states probed in three-terminal quantum dots, Phys. Rev. B 96, 195418 (2017).

[24] L. Tosi, C. Metzger, M. Goffman, C. Urbina, H. Pothier, S. Park, A. L. Yeyati, J. Nygård, and P. Krogstrup, SpinOrbit Splitting of Andreev States Revealed by Microwave Spectroscopy, Phys. Rev. X 9, 011010 (2019).

[25] C. Janvier, L. Tosi, L. Bretheau, Ç. Ö. Girit, M. Stern, P. Bertet, P. Joyez, D. Vion, D. Esteve, M. F. Goffman, H. Pothier, and C. Urbina, Coherent manipulation of andreev states in superconducting atomic contacts., Science 349, 1199 (2015).

[26] J. Klinovaja, S. Gangadharaiah, and D. Loss, ElectricField-Induced Majorana Fermions in Armchair Carbon Nanotubes, Phys. Rev. Lett. 108, 196804 (2012).

[27] J. D. Sau and S. Tewari, Topological superconducting state and majorana fermions in carbon nanotubes, Phys. Rev. B 88, 054503 (2013).

[28] M. Marganska, L. Milz, W. Izumida, C. Strunk, and M. Grifoni, Majorana quasiparticles in semiconducting carbon nanotubes, Phys. Rev. B 97, 075141 (2018).

[29] A. Kitaev, Fault-tolerant quantum computation by anyons, Ann. Phys. 303, 2 (2003).

[30] A. Kringhøj, L. Casparis, M. Hell, T. W. Larsen, F. Kuemmeth, M. Leijnse, K. Flensberg, P. Krogstrup, J. Nygård, K. D. Petersson, and C. M. Marcus, Anharmonicity of a superconducting qubit with a few-mode Josephson junction, Phys. Rev. B 97, 060508 (2018).

[31] J. R. Petta, A. C. Johnson, C. M. Marcus, M. P. Hanson, and A. C. Gossard, Manipulation of a Single Charge in a Double Quantum Dot, Phys. Rev. Lett. 93, 186802 (2004).

[32] Z. Penfold-Fitch, F. Sfigakis, and M. Buitelaar, Microwave Spectroscopy of a Carbon Nanotube Charge Qubit, Phys. Rev. Appl. 7, 054017 (2017).

[33] D. I. Schuster, A. Wallraff, A. Blais, L. Frunzio, R.-S. Huang, J. Majer, S. M. Girvin, and R. J. Schoelkopf, Ac Stark Shift and Dephasing of a Superconducting Qubit Strongly Coupled to a Cavity Field, Phys. Rev. Lett. 94, 123602 (2005).

[34] A. Abragam, The Principles of Nuclear Magnetism (Oxford University Press, London, 1961).

[35] A. Blais, R.-S. Huang, A. Wallraff, S. Girvin, and R. Schoelkopf, Cavity quantum electrodynamics for superconducting electrical circuits: An architecture for quantum computation, Phys. Rev. A 69, 062320 (2004).

[36] See Supplemental Material at http://link.aps.org/supple mental/10.1103/PhysRevApplied.15.064050 for data of additional devices, extracted coupling strengths, a derivation of qubit frequency and anharmonicity, a comparison of the coherence measurements and signatures of Rabi oscillations.

[37] J. Koch, T. M. Yu, J. Gambetta, A. A. Houck, D. I. Schuster, J. Majer, A. Blais, M. H. Devoret, S. M. Girvin, and R. J. Schoelkopf, Charge-insensitive qubit design derived from the cooper pair box, Phys. Rev. A 76, 042319 (2007).

[38] J.-P. Cleuziou, W. Wernsdorfer, V. Bouchiat, T. Ondarçuhu, and M. Monthioux, Carbon nanotube superconducting quantum interference device, Nat. Nanotechnol. 1, 53 (2006). 
[39] L. Bretheau, Ç. Ö. Girit, H. Pothier, D. Esteve, and C. Urbina, Exciting andreev pairs in a superconducting atomic contact, Nature 499, 312 (2013).

[40] Y.-J. Doh, J. A. van Dam, A. L. Roest, E. P. A. M. Bakkers, L. P. Kouwenhoven, and S. De Franceschi, Tunable supercurrent through semiconductor nanowires, Science 309, 272 (2005).

[41] A. A. Golubov, M. Y. Kupriyanov, and E. Il'ichev, The current-phase relation in josephson junctions, Rev. Mod. Phys. 76, 411 (2004).

[42] C. W. J. Beenakker, Universal Limit of Critical-Current Fluctuations in Mesoscopic Josephson Junctions, Phys. Rev. Lett. 67, 3836 (1991).

[43] C. W. J. Beenakker and H. van Houten, Josephson Current through a Superconducting Quantum Point Contact Shorter Than the Coherence Length, Phys. Rev. Lett. 66, 3056 (1991).

[44] M. Mergenthaler, F. J. Schupp, A. Nersisyan, N. Ares, A. Baumgartner, C. Schönenberger, G. A. D. Briggs, P. J. Leek, and E. A. Laird, Radio-frequency characterization of a supercurrent transistor made from a carbon nanotube, arXiv:103.16256 (2021).

[45] D. V. Averin, Coulomb Blockade in Superconducting Quantum Point Contacts, Phys. Rev. Lett. 82, 3685 (1999).

[46] A. Bargerbos, W. Uilhoorn, C.-K. Yang, P. Krogstrup, L. P. Kouwenhoven, G. de Lange, B. van Heck, and A. Kou, Observation of Vanishing Charge Dispersion of a Nearly Open Superconducting Island, Phys. Rev. Lett. 124, 246802 (2020).

[47] A. Wallraff, D. I. Schuster, A. Blais, L. Frunzio, J. Majer, M. H. Devoret, S. M. Girvin, and R. J. Schoelkopf, Approaching Unit Visibility for Control of a Superconducting Qubit with Dispersive Readout, Phys. Rev. Lett. 95, 060501 (2005).

[48] R. Vijay, C. Macklin, D. H. Slichter, S. J. Weber, K. W. Murch, R. Naik, A. N. Korotkov, and I. Siddiqi, Stabilizing Rabi oscillations in a superconducting qubit using quantum feedback, Nature 490, 77 (2012).
[49] A. D. O’Connell, M. Ansmann, R. C. Bialczak, M. Hofheinz, N. Katz, E. Lucero, C. McKenney, M. Neeley, H. Wang, E. M. Weig, A. N. Cleland, and J. M. Martinis, Microwave dielectric loss at single photon energies and millikelvin temperatures, Appl. Phys. Lett. 92, 112903 (2008).

[50] R. Vijay, J. D. Sau, M. L. Cohen, and I. Siddiqi, Optimizing Anharmonicity in Nanoscale Weak Link Josephson Junction Oscillators, Phys. Rev. Lett. 103, 087003 (2009).

[51] R. Vijay, E. M. Levenson-Falk, D. H. Slichter, and I. Siddiqi, Approaching ideal weak link behavior with three dimensional aluminum nanobridges, Appl. Phys. Lett. 96, 223112 (2010).

[52] E. M. Levenson-Falk, R. Vijay, and I. Siddiqi, Nonlinear microwave response of aluminum weak-link josephson oscillators, Appl. Phys. Lett. 98, 123115 (2011).

[53] A. Zazunov, V. S. Shumeiko, G. Wendin, and E. N. Bratus', Dynamics and phonon-induced decoherence of andreev level qubit, Phys. Rev. B 71, 214505 (2005).

[54] J. Gramich, A. Baumgartner, and C. Schönenberger, Resonant and Inelastic Andreev Tunneling Observed on a Carbon Nanotube Quantum Dot, Phys. Rev. Lett. 115, 216801 (2015).

[55] B. H. Schneider, S. Etaki, H. S. J. van der Zant, and G. A. Steele, Coupling carbon nanotube mechanics to a superconducting circuit, Sci. Rep. 2, 599 (2012).

[56] L. Casparis, T. Larsen, M. Olsen, F. Kuemmeth, P. Krogstrup, J. Nygård, K. Petersson, and C. Marcus, Gatemon Benchmarking and Two-Qubit Operations, Phys. Rev. Lett. 116, 150505 (2016).

[57] J. Kelly et al., State preservation by repetitive error detection in a superconducting quantum circuit, Nature 519, 66 (2015).

[58] J. Moser, J. Güttinger, A. Eichler, M. J. Esplandiu, D. E. Liu, M. I. Dykman, and A. Bachtold, Ultrasensitive force detection with a nanotube mechanical resonator, Nat. Nanotechnol. 8, 493 (2013). 